\title{
BurstCube: A CubeSat for Gravitational Wave Counterparts
}

\author{
Judith Racusin* \\ $N A S A / G S F C$ \\ E-mail: judith.racusin@nasa.gov
}

\section{Jeremy S. Perkins}

$N A S A / G S F C$

E-mail: jeremy.s.perkins@nasa.gov

Michael S. Briggs ${ }^{a}$, Georgia de Nolfo $^{b}$, John Krizmanic ${ }^{c}$, Regina Caputo ${ }^{c}$, Julie E. McEnery $^{b}$, Peter Shawhan ${ }^{d}$, David Morris ${ }^{e}$, Valerie Connaughton ${ }^{f}$, Dan Kocevski ${ }^{g}$, Colleen Wilson-Hodge ${ }^{g}$, Michelle Hui $^{g}$, Lee Mitchell ${ }^{h}$, \& Sheila McBreen ${ }^{i}$

${ }^{a}$ University of Alabama, Huntsville

${ }^{b} N A S A / G S F C$

${ }^{c} N A S A / G S F C / C R E S S T$

${ }^{d}$ University of Maryland, College Park

${ }^{e}$ University of the Virgin Islands

${ }^{f}$ USRA

${ }^{g} N A S A / M S F C$

${ }^{h}$ Naval Research Laboratory

${ }^{i}$ University College Dublin

We present BurstCube, a novel CubeSat that will detect and localize Gamma-ray Bursts (GRBs). BurstCube will detect long GRBs, attributed to the collapse of massive stars, short GRBs (sGRBs), resulting from binary neutron star mergers, as well as other gamma-ray transients in the energy range 10-1000 keV. sGRBs are of particular interest because they are predicted to be the counterparts of gravitational wave $(\mathrm{GW})$ sources soon to be detectable by LIGO/Virgo. BurstCube contains 4 CsI scintillators coupled with arrays of compact low-power Silicon photomultipliers (SiPMs) on a 6U Dellingr bus, a flagship modular platform that is easily modifiable for a variety of $6 \mathrm{U}$ CubeSat architectures. BurstCube will complement existing facilities such as Swift and Fermi in the short term, and provide a means for GRB detection, localization, and characterization in the interim time before the next generation future gamma-ray mission flies, as well as space-qualify SiPMs and test technologies for future use on larger gamma-ray missions. The ultimate configuration of BurstCube is to have a set of $\sim 10$ BurstCubes to provide all-sky coverage to GRBs for substantially lower cost than a full-scale mission.

35th International Cosmic Ray Conference - ICRC2017

10-20 July, 2017

Bexco, Busan, Korea

${ }^{*}$ Speaker. 


\section{Introduction}

The first direct detections of Gravitational Waves (GWs) by the Laser Interferometer GW Observatory (LIGO) of GW150914, GW151226 and GW170104, the mergers of stellar-mass black hole $(\mathrm{BH})$ binaries $[1,2,3]$, has brought $\mathrm{GW}$ astronomy into a new era of discovery. The search for electromagnetic (EM) counterparts to GW sources is now more important than ever before, in order to provide astrophysical context, and validate low-significance signals. Prior to the discovery of GW150914, most of the theoretical predictions for EM counterparts to GW sources were for compact object mergers involving a neutron star (NS; i.e. NS-NS and NS-BH). However, a candidate $\gamma$-ray counterpart that is both temporally and spatially coincident with GW150914 was detected by the Fermi Gamma-ray Burst Monitor (GBM) [4], and is consistent with being a low-fluence short $\gamma$-ray burst (sGRB), providing new hope for detecting EM counterparts to BH-BH mergers $[5,6,7,8,9]$.

LIGO and Virgo are commissioning major upgrades to reach new design sensitivities by 2019, adding the detectability of GWs from the inspiral of systems including a NS, generally believed to be the progenitors of sGRBs [10]. The simultaneous discovery of GW and EM signatures requires dedicated and coordinated observations by large communities of both ground and space-based observatories. Existing sensitive $\gamma$-ray burst (GRB) observatories cover only $\sim 70 \%$ of the sky at any one time, and any increase in sky coverage by additional facilities increases both the likelihood of coincident detection, and the number of sGRBs that can be correlated with GW signals.

The recent detection of GW signals from binary BH mergers is the most important discovery of this decade so far. It may be surpassed in the coming years by further GW discoveries, especially NS binary mergers which may reveal the mechanisms of most sGRBs and nuclear astrophysics. The GBM candidate coincident sGRB only adds to the need for EM searches for GW counterparts. The Advanced LIGO and Advanced Virgo GW observatories (hereafter LIGO/Virgo) are scheduled to reach design sensitivity in 2019, with LIGO-India to be added in 2022 [10]. The current fleet of sensitive spaceborne GRB instruments with localization capability has incomplete sky coverage and uncertain operation beyond 2019. Coincident detection of sGRBs and GW sources will:

- provide the "smoking gun" evidence for the progenitors of sGRBs for the first time;

- increase the confidence in low-significance LIGO/Virgo GW detections;

- provide small temporal and positional windows for targeted searches of GW data that will enable detecting weaker GW signals than can be detected in blind searches lacking priors, increasing the search volume;

- provide the astrophysical context for the GW signal via population statistics on jet beaming angles and $\gamma$-ray energetics as inputs into stellar population synthesis models;

- provide localizations that will assist wide-field follow-up observers in afterglow detection and redshift measurement which will lead to insight into cosmological parameter estimation, constraints on the NS equation of state [11], and an inventory of r-process elements in the Universe constrained by the faint sGRB kilonova signature [12]. 


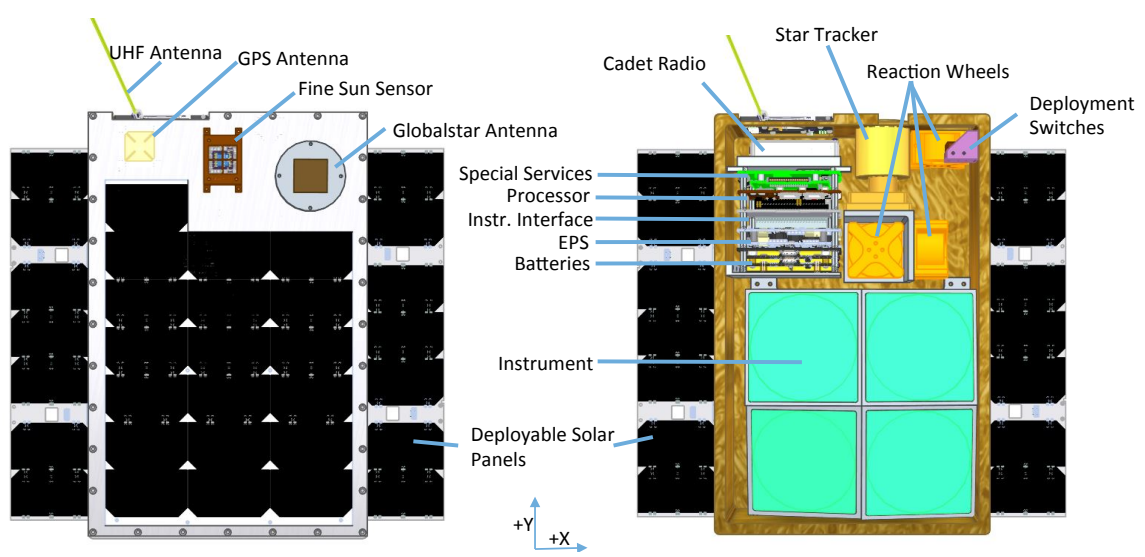

Figure 1: Internal view of BurstCube instrument and spacecraft components (right). BurstCube bodymounted and deployable solar panels (left).

\section{BurstCube}

Over the past decade there has been a renaissance in the development of small-sat and CubeSat technologies. The result is that it is achievable to build a CubeSat to detect the bright signals from GRBs. These proceedings detail a 6U CubeSat concept called BurstCube based on the Dellingr platform developed at GSFC but the overall concept can be generalized and use either a custom or commercial bus and be larger or smaller than BurstCube. The main limiting factors in developing a mission like BurstCube are mass, power, and volume but the advent of low-power, low-volume SiPMs enables a workable detector readout design. We describe the BurstCube CubeSat, which provides rapidly available high-resolution temporal, spectral, and localization data. Even rough localizations will be helpful to verify weak GW signals, as the temporal coincidence is the most important measurement alerting the community to a potential on-axis event. BurstCube increases the sky coverage beyond existing facilities, and is optimized for detecting sGRBs, all for a small fraction of the cost of a larger mission.

BurstCube is a $6 \mathrm{U}$ CubeSat divided into a $4 \mathrm{U}$ instrument package, and $2 \mathrm{U}$ of spacecraft subsystems (Fig. 1). The instrument concept is similar to Fermi-GBM [13], except BurstCube uses $\mathrm{CsI}$ as a primary detection medium covering the energy range from $10 \mathrm{keV}-1 \mathrm{MeV}$ with high efficiency and adequate energy resolution. These crystals are inexpensive and have a long track record of use in $\gamma$-ray astronomy. A design philosophy is to rely on proven well-tested technology.

Each of the four detectors are $9.4 \times 9.4 \times 1.27 \mathrm{~cm}$, with the maximum dimension dictated by the size of the $6 \mathrm{U}$ CubeSat. The detectors are composed of CsI crystals viewed by an array of low-power and low-voltage (20-70 V) Silicon Photomultipliers (SiPMs). Compared the more conventional photomultiplier tubes, SiPMs significantly reduce mass, volume, power, and cost. The combination of scintillation crystals and new readout devices makes it possible to consider a compact, low-power instrument that is readily deployable on a CubeSat platform. The format of the $6 \mathrm{U}$ spacecraft drives the instrument mass, power, and volume budget. However, even in this compact design the detector presented here is competitive with the state of the art system (Fermi-GBM). The BurstCube team is exploring a modification to the baseline design by further segmenting the detector system and triggering over summed detectors to better localize bright GRBs.

To estimate the BurstCube effective area and resulting sensitivity, we simulated a single CsI detector using MEGAlib [14] (which provides an interface to GEANT4). The simulated BurstCube 


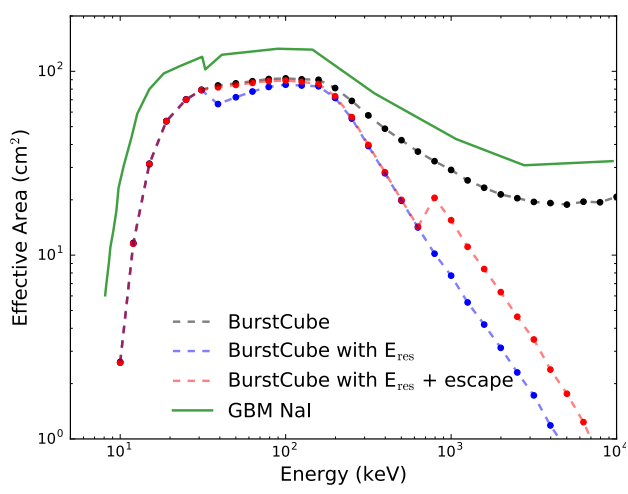

Figure 2: Despite the constraints of a CubeSat, BurstCube achieves an effective area of $70 \%$ of GBM at $100 \mathrm{keV}$ and $15^{\circ}$ incidence The effective area as a function of energy, and the corresponding curve for the larger GBM NaI detectors are shown for reference.

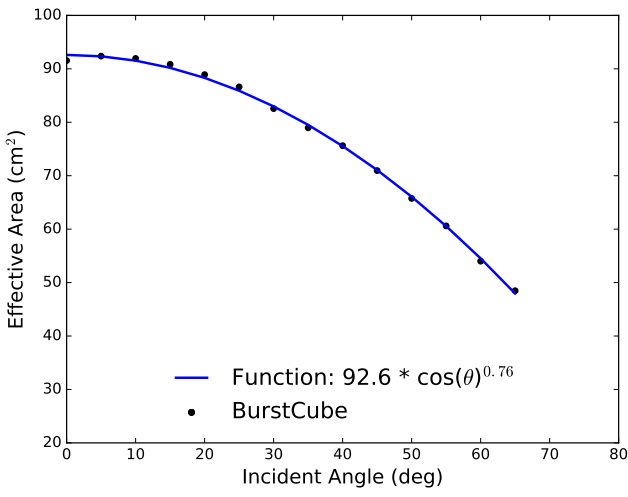

Figure 3: The square BurstCube detectors make maximum use of space and still achieve an effective area cosine dependence on incidence angle expected for a thin detector which is needed for GRB localizations.

effective area is competitive with GBM (Fig. 2), despite the smaller detectors.

BurstCube will localize GRBs in a similar manner to BATSE or GBM [15]. The approximate cosine dependence $\left(\sim \cos (\theta)^{0.76}\right)$ of the effective area with incidence angle $(\mathbf{F i g} . \mathbf{3})$ is leveraged to localize GRBs by measuring the relative brightness of the burst in each detector. The detector count rates will be matched to a previously computed table of relative detector responses as a function of spacecraft coordinates. Depending on the region of the sky in which the burst occurs, the localization accuracy can be as good as a few degrees or as high as 90 degrees. The localization accuracy for each position on the sky in Azimuth and Elevation coordinates (Figs. 4 and 5), shows that over the $\sim 1 / 3$ of the sky seen with 3 or 4 detectors, localizations have an typical accuracy of $<7^{\circ}$ radius.

BurstCube will detect $\gtrsim 24$ sGRBs per year, in addition to $>100$ long GRBs and other transients. GRB rate estimates are made by scaling the GBM GRB detections [16] to BurstCube based upon comparison between simulations of both the BurstCube and GBM NaI detector systems. We estimate the BurstCube limiting flux for the various trigger timescales in the $50-300 \mathrm{keV}$

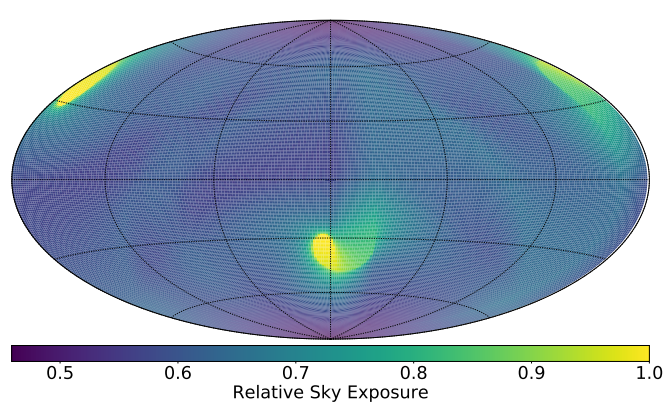

Figure 4: The integrated relative exposure of BurstCube over a single orbit projected onto the sky in Mollweide celestial coordinates. An exposure of 1 is the maximum on-axis exposure for a single CsI detector.

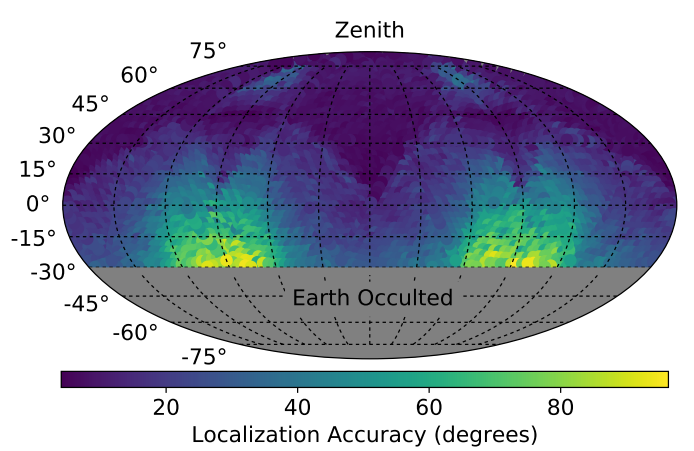

Figure 5: BurstCube localizes sGRBs to an accuracy of $\sim 7^{\circ}$ radius when viewed by 3 or more detectors. The localization errors (in degrees radius) are shown across the entire BurstCube FoV in Az/El coordinates. 
band, and compare those to the similar GBM distributions to determine which GBM sGRBs would be detectable by BurstCube. These calculations use only a few of the most common trigger criteria from GBM, likely underestimating the sGRB rate by $20-30 \%$ due to not including the full range of energies and timescales in the robust GBM triggering flight software.

The BurstCube instrument is housed in a CubeSat bus based on the Dellingr platform, a modular platform designed to be easily modifiable for a variety of $6 \mathrm{U}$ CubeSat architectures (see Fig. 1). It includes 3 axis-stabilized pointing control, Ultra-High Frequency (UHF) communication system, body mounted solar panels, and is designed for the Planetary Systems Corporation (PSC) 6U canister standard ${ }^{1}$. The Dellingr bus will be enhanced this year to include deployable solar panels and a star tracker. The only additional system needed for BurstCube is the Globalstar communications system for rapid GRB location distribution.

\section{Performance}

The expected rate of coincident GW sources with SGRB detections with BurstCube is $\sim 2.2 \mathrm{yr}^{-1}$ for NS-NS progenitors, and potentially significantly higher if SGRB progenitors include a BH. This estimate is drawn entirely from the observed sGRB rate, the GW detection range, and the intrinsic rate density of sGRBs in the nearby Universe and on the sensitivities of the detectors, but depends only weakly (quadratically) on the poorly-constrained beaming angle of sGRBs [17]. BurstCube and other GRB-detecting observatories enhance the LIGO/Virgo detection rate by enabling the detection of weaker GW signals due to their temporal and positional correlation with detected sGRBs. GW/sGRB rates are estimated by multiplying the search volume by an $o b$ served sGRB rate of $10 \pm 5 \mathrm{Gpc}^{-3} \mathrm{yr}^{-1}[18,19]$. The search volume depends upon the makeup of the progenitor system, as the strength of the GW signal scales with the mass of the system, making binaries containing a $\mathrm{BH}$ detectable by LIGO/Virgo to larger distances. A NS-BH binary increases the detection range compared to a NS-NS by a factor of $\sim 1.6$, and $\mathrm{BH}-\mathrm{BH}$ by a factor of $2-5$ [20]. A factor of 1.5 is added due to the preferential enhancement of the GW signal along the jet axis, and another factor of 1.5 due to sGRB triggers seeding sub-threshold GW searches. If sGRBs are also due to NS-BH mergers or BH-BH mergers, the $\mathrm{SGRB} / \mathrm{GW}$ rate would increase by a factor of a few to hundreds. BurstCube is sensitive enough to detect the $\gamma$ rays from any sGRB close enough for the GWs to be detectable by LIGO/Virgo.

Because of the low expected rate of joint GW/sGRB detections, it is crucial to detect sGRBs from the entire sky at all times. Failure to do so will lead to a very small sample of GW/sGRB coincident observations, greatly weakening the scientific return of LIGO/Virgo. BurstCube is designed as a low cost method to maximize sky coverage for detecting sGRBs to correlate with LIGO/Virgo.

BurstCube complements existing and future GRB instruments by increasing the probability of coincident GW/sGRB detections, by increasing sky coverage. GBM is the current best GRB instrument for supporting the GW detectors, due to its very wide field-of-view (FoV) and excellent detection sensitivity for sGRBs with its $12 \mathrm{NaI}$ and $2 \mathrm{BGO}$ detectors [13]. However, any similar observatory in a low Earth orbit can operate with at most $\sim 60 \%$ duty cycle for any point on the

\footnotetext{
${ }^{1}$ Also compatible with the NanoRacks $6 \mathrm{U}$ dispenser
} 
sky due to Earth blockage and South Atlantic Anomaly (SAA) passage. In comparison, Swift-BAT [21] views $\sim 20 \%$ of the sky at any one time, and the approved SVOM mission [22] has limited

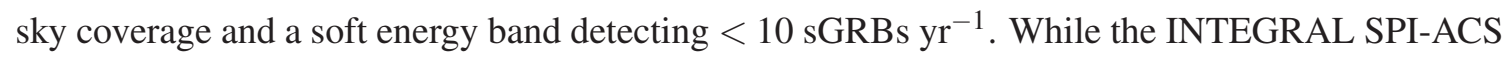
provides all-sky observations, it cannot localize or provide spectral information. The Interplanetary Network (IPN) provides approximately all-sky coverage for bright sGRBs, but cannot probe fainter bursts, and has a data latency of up to several days. BurstCube will increase sky exposure by $\sim 15 \%$ on average depending on orbit phasing. The need for this is clear; even with the large FoVs of BAT and GBM the error contour of GW150914 was not fully covered at trigger [23, 4, 24]. Even if Swift and Fermi continue to be operational in 2020, exposure improves with BurstCube as it provides a cost effective and timely method to support and enhance LIGO/Virgo science as they reach design sensitivity.

While both LIGO/Virgo and BurstCube localizations on their own are 10's to 100's of $\mathrm{deg}^{2}$, the intersection of GW and BurstCube localizations (Fig. 6) for coincident detections will reduce the area to be more manageable for tiling. The reduction of these error regions will be especially important for events seen by only two GW detectors, sub-threshold GW signals, and those beyond the Virgo horizon.

BurstCube will complement other observatories by:

- providing higher energy $\gamma$-ray coverage than Swift-BAT (15-150 keV) that can be used in tandem to understand prompt emission spectra and estimate energetics;

- providing rapid ( $\lesssim 5$ minutes) localizations distributed via the Gamma-ray Coordinates Network (GCN) for afterglow searches by wide-FoV ground-based optical and space-based Xray telescopes like ZTF [25], MASTER [26] and TAO-ISS (proposed) [27];

- adding to the IPN producing time-of-arrival localizations over long baselines.

BurstCube will also be sensitive to soft $\gamma$-ray phenomena including soft $\gamma$-ray repeaters, solar flares, and other transients.

As a CubeSat, the BurstCube detector sizes and quantity are limited compared to GBM. GBM has a significantly wider energy range $(8 \mathrm{keV}-30 \mathrm{MeV})$ due to the inclusion of two BGO detectors, each of which is more massive than a $6 \mathrm{U}$ CubeSat. However, several improvements compared to GBM ameliorate the size and number limitation. The use of CsI instead of NaI provides better efficiency and more sensitivity at higher energies per unit detector volume. The smaller spacecraft will cause less scattering of $\gamma$ rays, and that scattering will be easier to model, reducing systematics in localizations. Zenith (or Sun) pointing instead of the $50^{\circ}$ rocking of Fermi will simplify the

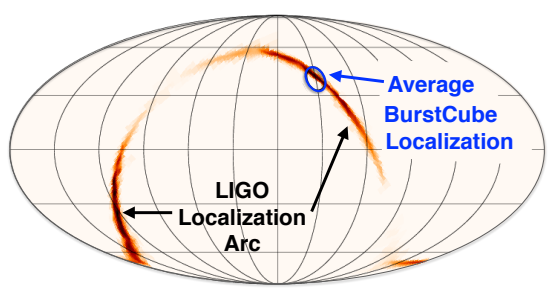

Figure 6: In some regions of the sky, the conjunction of BurstCube and LIGO/Virgo localizations will reduce the followup search area, especially in cases when the GW event is only seen by 2 detectors, as demonstrated by overlaying a simulated BurstCube localization over the LIGO localization map of GW151226 [2]. The typical BurstCube localization (blue circle) is $\sim 7^{\circ}$ radius for the $2 / 3$ of the unocculted sky with 3 or more detector exposures overlapping. 
modeling of $\gamma$ rays scattered by the Earth. The BurstCube detectors will have passive shielding to further reduce the effects of scattered $\gamma$ rays. A faster on board computer will allow a more sophisticated triggering algorithm optimized for sGRBs by using pre- and post-source background intervals.
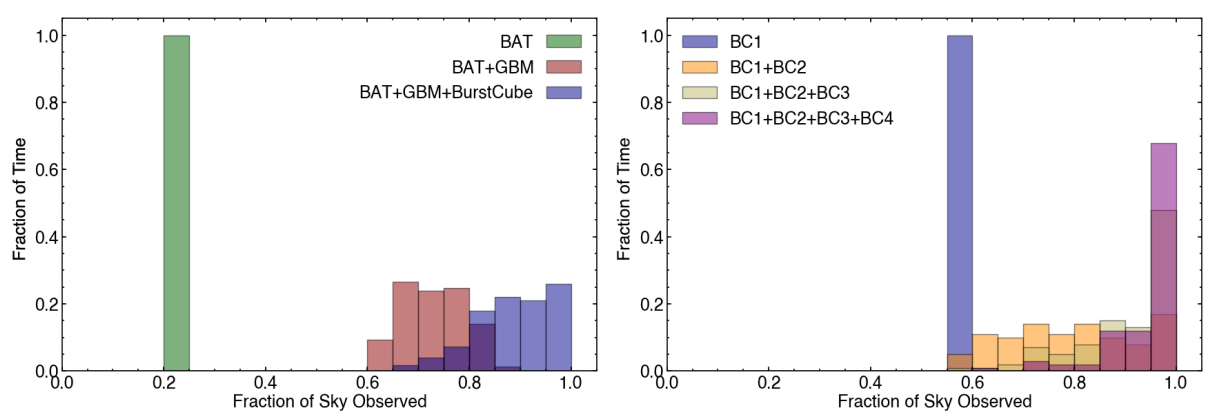

Figure 7: Adding BurstCube to the existing network of GRB instruments (left) will increase sky coverage to improve the probability of detecting and characterizing coincident emission from a sGRB and GW trigger. A set of 3 or more BurstCubes (right) would also provide all-sky coverage.

Accounting for the orbit phasing of BurstCube (assuming an ISS orbit), Fermi, and Swift, BurstCube increases the average sky coverage from $\sim 70 \%$ to $~ 90 \%$ (Fig. 7). When LIGO/Virgo reach full sensitivity and joint GW/sGRB detections become a realistic prospect, it is vital that detectors sensitive to sGRBs with broad sky coverage be operational.

\section{References}

[1] B. P. Abbott et al., Observation of Gravitational Waves from a Binary Black Hole Merger, Physical Review Letters 116 (Feb., 2016) 061102, [1602 .03837].

[2] B. P. Abbott et al., GW151226: Observation of Gravitational Waves from a 22-Solar-Mass Binary Black Hole Coalescence, Physical Review Letters 116 (June, 2016) 241103, [1606. 04855$].$

[3] Ligo Scientific and Virgo Collaboration collaboration, B. P. Abbott, R. Abbott, T. D. Abbott, F. Acernese, K. Ackley, C. Adams et al., Gw170104: Observation of a 50-solar-mass binary black hole coalescence at redshift 0.2, Phys. Rev. Lett. 118 (Jun, 2017) 221101.

[4] V. Connaughton et al., Fermi GBM Observations of LIGO Gravitational Wave event GW150914, ArXiv:1602.03920 (Feb., 2016) , [1602.03920].

[5] B. Zhang, Possible Short Gamma-Ray Bursts Associated with Black Hole - Black Hole Mergers, ArXiv:1602.04542 (Feb., 2016) , [1602.04542].

[6] X. Li, F.-W. Zhang, Q. Yuan, Z.-P. Jin, Y.-Z. Fan, S.-M. Liu et al., Implication of the association between GBM transient 150914 and LIGO Gravitational Wave event GW150914, ArXiv:1602.04460 (Feb., 2016), [1602.04460].

[7] A. Loeb, Electromagnetic Counterparts to Black Hole Mergers Detected by LIGO, ArXiv:1602.04735 (Feb., 2016) , [1602.04735].

[8] R. Yamazaki, K. Asano and Y. Ohira, Electromagnetic Afterglows Associated with Gamma-Ray Emission Coincident with Binary Black Hole Merger Event GW150914, ArXiv:1602.05050 (Feb., 2016), [1602.05050]. 
[9] R. Perna, D. Lazzati and B. Giacomazzo, Short Gamma-Ray Bursts from the Merger of Two Black Holes, ArXiv:1602.05140 (Feb., 2016) , [1602.05140].

[10] B. P. Abbott et al., Prospects for Observing and Localizing Gravitational-Wave Transients with Advanced LIGO and Advanced Virgo, Living Reviews in Relativity 19 (Dec., 2016) 1, [1304.0670].

[11] W. Del Pozzo et al., Demonstrating the Feasibility of Probing the Neutron-Star Equation of State with Second-Generation Gravitational-Wave Detectors, Physical Review Letters 111 (Aug., 2013) 071101, [1307.8338].

[12] B. D. Metzger et al., Electromagnetic counterparts of compact object mergers powered by the radioactive decay of r-process nuclei, MNRAS 406 (Aug., 2010) 2650-2662, [1 001.5029 ].

[13] C. Meegan et al.ApJ 702 (Sept., 2009) 791-804, [0 908 . 0450 ].

[14] A. Zoglauer, R. Andritschke and F. Schopper, MEGAlib The Medium Energy Gamma-ray Astronomy Library, .

[15] G. J. Fishman et al., Overview of Observations from BATSE on the Compton Observatory, A\&AS 97 (Jan., 1993) 17.

[16] A. von Kienlin et al., The Second Fermi GBM Gamma-Ray Burst Catalog: The First Four Years, ApJS 211 (Mar., 2014) 13, [1401.5080].

[17] W. Fong et al., A Jet Break in the X-Ray Light Curve of Short GRB 111020A: Implications for Energetics and Rates, ApJ 756 (Sept., 2012) 189, [1204.5475].

[18] D. Guetta and T. Piran, The batse-swift luminosity and redshift distributions of short-duration grbs, A\&A 453 (2006) 823-828.

[19] D. M. Coward et al., The Swift short gamma-ray burst rate density: implications for binary neutron star merger rates, MNRAS 425 (Oct., 2012) 2668-2673, [1202 . 2179].

[20] B. P. Abbott et al., The Rate of Binary Black Hole Mergers Inferred from Advanced LIGO Observations Surrounding GW150914, ArXiv:1602.03842 (Feb., 2016) , [1602.03842].

[21] S. D. Barthelmy et al., The Burst Alert Telescope (BAT) on the SWIFT Midex Mission, Space Science Reviews 120 (Oct., 2005) 143-164, [astro-ph/0507410].

[22] D. Götz and SVOM Collaboration, SVOM: a new mission for Gamma-Ray Bursts studies , Memorie della Societa Astronomica Italiana Supplementi 21 (2012) 162.

[23] B. P. Abbott et al., Localization and broadband follow-up of the gravitational-wave transient GW150914, ArXiv e-prints (Feb., 2016), [1602.08492].

[24] P. A. Evans et al., Swift follow-up of the Gravitational Wave source GW150914, ArXiv e-prints (Feb., 2016), [1602.03868].

[25] E. Bellm, The Zwicky Transient Facility, in The Third Hot-wiring the Transient Universe Workshop (P. R. Wozniak, M. J. Graham, A. A. Mahabal and R. Seaman, eds.), pp. 27-33, 2014, 1410.8185.

[26] V. M. Lipunov et al., MASTER: The Mobile Astronomical System of Telescope-Robots, Astronomische Nachrichten 325 (Oct., 2004) 580-582, [a stro-ph/ 0411757 ].

[27] J. Camp et al., Using ISS telescopes for electromagnetic follow-up of gravitational wave detections of NS-NS and NS-BH mergers, Experimental Astronomy 36 (Dec., 2013) 505-522, [1304 . 3705 ]. 